\title{
PREDITORES EMOCIONAIS E SOCIAIS DA ACEITAÇÃO PELOS PARES EM CRIANÇAS DE IDADE ESCOLAR
}

\author{
Diana Rute Pereira Alves ${ }^{1}$ \\ Orlanda Cruz ${ }^{2}$
}

Resumo: Este estudo pretende contribuir para uma visão compreensiva dos processos emocionais e sociais no domínio das relações entre pares. Mais especificamente, trata-se de estudar a capacidade preditiva do conhecimento emocional e das habilidades sociais na determinação da aceitação pelos pares. Participaram neste estudo, 131 crianças que frequentavam o $3^{\circ}$ ano de escolaridade. Os resultados revelaram que as habilidades sociais medeiam o efeito do conhecimento emocional na aceitação pelos pares. O contributo do conhecimento emocional da criança para a sua aceitação pelos pares é explicado de forma significativa pelas suas habilidades sociais.

Palavras-chave: conhecimento emocional, habilidades sociais, aceitação pelos pares

Emotional and social predictors of peer acceptance in school-age children (Abstract): This study intends to contribute to a comprehensive vision of emotional and social processes in peer relations. It is intended to analyse the role of emotion knowledge and social abilities as predictors of peer acceptance. The participants were 131 children attending third grade, aged eight to nine years. The results showed that social abilities mediated the effect of emotion knowledge on peer acceptance. The effect of child emotional knowledge on peer acceptance was largely explained by her/his social abilities.

Keywords: emotion knowledge, social abilities, peer acceptance

\footnotetext{
${ }^{1}$ Universidade do Porto, Faculdade de Psicologia e de Ciências da Educação

2 Universidade do Porto, Faculdade de Psicologia e de Ciências da Educação

Correspondência: Diana Alves, Faculdade de Psicologia e de Ciências da Educação da Universidade do Porto, Rua Dr. Manuel Pereira da Silva; 4200-392 Porto, Portugal. Email: dianalves@fpce.up.pt; Tel.: 00351 226079700; Fax: 00351226079725

O presente estudo está integrado num projecto de investigação mais amplo sobre o "Desenvolvimento das Competências Sociais de Crianças em Idade Escolar", financiado pela Fundação para Ciência e Tecnologia (FCT), através do Centro de Psicologia da Faculdade do Porto.
} 


\section{Introdução}

Uma das tarefas centrais da criança em idade escolar consiste no desenvolvimento de aptidões sociais que promovam a construção e a manutenção de relações sociais gratificantes com companheiros de idades aproximadas. As relações com pares são contextos relacionais promotores do desenvolvimento e aprendizagem de múltiplas capacidades sociais, emocionais e cognitivas, determinantes do ajustamento social. A aceitação pelos pares, definida em função das preferências e rejeições que a criança recebe no seu grupo de pares, é uma medida frequentemente utilizada como indicador do funcionamento social da criança. As crianças com melhores níveis de aceitação pelos pares tendem a apresentar melhor ajustamento escolar (Izard, Fine, Schultz, Mostow, Ackerman \& Youngstrom, 2001; Machado, Veríssimo, Torres, Peceguina, Santos \& Rolão, 2008) e relações de amizade mais estáveis (Terry, 2002). Inúmeros estudos sociométricos evidenciam a aceitação pelos pares como um dos indicadores mais fiáveis do ajustamento psicológico na adolescência e na idade adulta (Zettergren, 2005).

Nas últimas décadas os investigadores têm vindo a estudar os preditores da aceitação pelos pares, nomeadamente as competências sociais e o conhecimento emocional (Denhan, McKinley, Couchoud \& Holt, 1990; Eisenberg \& Fabes, 1998). A investigação que relatamos neste artigo pretende ser um contributo para o estudo destes preditores. Apresenta-se de seguida uma síntese da revisão da literatura que suporta a importância das variáveis sociais e emocionais na determinação da aceitação pelos pares.

As crianças populares tendem a ser avaliadas como mais agradáveis e cooperantes, menos agressivas e difíceis (Denham \& Holt, 1993); e tendem a manifestar mais comportamentos assertivos e de liderança (Dodge \& Feldman, 1990). Pelo contrário, as crianças rejeitadas caracterizam-se por apresentarem mais frequentemente comportamentos agressivos e de violação de regras, hiperactividade (Coie, Dodge \& Kupersmidt, 1990; Rubin, Bukowski \& Parker, 2006) e ausência de comportamentos pró-sociais (Dodge, 1983).

As respostas sociais manifestas pela criança nas suas interacções sociais reflectem o modo como processa os estímulos sociais, os quais assumem a forma de problemas ou dilemas a resolver nessas interacções (Almeida, 1997). O Modelo do Processamento da Informação Social (Crick \& Dodge, 1994) permite uma visão articulada das estratégias de resolução de problemas sociais, utilizadas pelas crianças nas suas interacções sociais com pares. De acordo com este modelo, quando confrontadas com problemas de interacção social, as crianças avançam ao longo de seis etapas: (1) codifica- 
ção da informação recebida (e.g., comportamento de agressão por parte de um colega), (2) interpretação dessa informação (e.g., trata-se ou não de um comportamento intencional), (3) clarificação dos objectivos (e.g., face à informação e interpretação do comportamento do colega, a manutenção da relação com este é ou não um objectivo), (4) acesso ou construção de respostas alternativas (e.g., retaliar a agressão ou ignorá-la), (5) avaliação de cada uma das respostas alternativas e tomada de decisão quanto ao comportamento a adoptar (ou seja, avaliação das consequências em termos de probabilidade de sucesso de cada alternativa face aos objectivos clarificados anteriormente) e, finalmente, (6) actuação comportamental. A forma como os pares avaliam esta resposta e lhe reagem funciona como um novo estímulo social a ser processado subsequentemente. O processamento da informação social apresenta variações interindividuais em função dos dados armazenados naquilo que Crick e Dodge (1994) denominam data base: as predisposições temperamentais e as experiências anteriormente vivenciadas. Dada a constância das interacções sociais no dia-a-dia das crianças, esta base de dados apresenta-se em contínua actualização.

Uma característica fundamental das interacções sociais é a sua forte conotação emocional, pelo que se torna imprescindível considerar o papel das emoções no processamento da informação social (Lemerise \& Arsenio, 2000). Considerando o Modelo do Processamento da Informação Social atrás apresentado, as emoções estão presentes em qualquer uma das etapas (codificação, interpretação clarificação de objectivos, alternativas de resposta, tomada de decisão e actuação comportamental), já que a informação processada não é apenas social, mas simultaneamente emocional (Lemerise \& Arsenio, 2000). Da mesma forma, a base de dados integra predisposições temperamentais, não só em termos sociais mas também em termos emocionais, nomeadamente a dimensão emocionalidade (Eisenberg, Murphy \& Shepard, 1997), e as experiências sociais anteriormente vivenciadas integram uma componente emocional (Arsenio \& Lover, 1995).

A capacidade de identificação e de nomeação das expressões emocionais, designada por Izard e Ackerman (2004) como conhecimento emocional, permite uma maior acuidade na identificação e interpretação dos sinais sociais (Lemerise \& Arsenio, 2000), e prediz a aceitação pelos pares (Mostow et al., 2002). A qualidade do relacionamento com pares pode ser potenciada de forma significativa pela utilização apropriada do conhecimento emocional. O conhecimento emocional fornece as bases necessárias à comunicação emocional inerente às relações sociais (Hobson, 1993; Izard, 1971), possibilitando uma apreensão adequada dos sinais sociais, facilitadora de comportamentos sociais adaptados e promotores da aceitação pelos pares, e activadora de emoções adequadas à continuidade das interacções (Izard, 1991, 1993). 
A leitura adequada das pistas emocionais em situações de conflito, a habilidade para reconhecer e lidar com as expressões emocionais, e a capacidade para identificar as causas e as consequências lógicas das emoções, são algumas das facetas do conhecimento emocional que podem predizer a aceitação pelos pares (Cassidy, Parke, Dutkovsky \& Braungart, 1992). Estudos desenvolvidos no âmbito do modelo do processamento da informação social (Crick \& Dodge, 1994; Schultz, Izard \& Bear, 2004) revelam que as crianças rejeitadas pelos pares são menos competentes na codificação e na interpretação das pistas sociais (Dodge \& Feldman, 1990), na regulação emocional e na expressão comportamental das emoções (Eisenberg et al., 1997). Ao ser rejeitada pelos pares, é possível que a criança se sinta sozinha e zangada, e estas emoções poderão levar a um enviesamento nas atribuições relativas às intenções dos pares, o que, por sua vez, pode predispor a criança para mais respostas agressivas e de retaliação. Podemos assim explicar a rejeição pelo enviesamento atribucional que esta causa no processamento da informação social (Dodge, Lansford, Burks, Bates, Pettit, Fontaine \& Price, 2003).

Alguns estudos mostram que a relação entre o conhecimento emocional e a aceitação pelos pares é mediada pelo comportamento social, ou seja, as interacções sociais funcionam como processos 'proximais' através dos quais o conhecimento emocional afecta a aceitação pelos pares (Arsenio, Cooperman \& Lover, 2000; Mostow et al., 2002). No seguimento destes estudos, pretende-se aqui analisar a capacidade preditiva das habilidades sociais e do conhecimento emocional na determinação da qualidade da relação com pares nas crianças em idade escolar, colocando a hipótese de as habilidades sociais mediarem a relação entre o conhecimento emocional e a aceitação pelos pares.

Atendendo a que a investigação sugere que as raparigas apresentam valores superiores de conhecimento emocional (Schultz, Izard \& Ackerman, 2000), por um lado, e de competências pró-sociais (Denhan, 1998; Eisenberg \& Fabes, 1998) por outro, a variável sexo será analisada. Da mesma maneira, existe alguma evidência na literatura acerca da importância do nível socioeconómico da família na determinação do conhecimento emocional da criança (Fine, Izard, Mostow, Trentacosta \& Ackerman, 2003) e das suas habilidades sociais (Hoff, Laursen \& Tardif, 2002), pelo que esta variável será também contemplada nas análises.

\section{Método}

\section{Participantes}

Participaram neste estudo 131 crianças (58 raparigas e 73 rapazes), com idades compreendidas entre os 8 e os 10 anos $(M=8,48 ; D P=0,55)$, que 
frequentavam oito escolas do $1^{\circ}$ ciclo do Ensino Básico na zona do Grande Porto. Atendendo à temática central e aos objectivos deste estudo, foram definidos os seguintes critérios de inclusão dos participantes na amostra: (1) frequência do $3^{\circ}$ ano de escolaridade, (2) não mais do que uma retenção escolar, (3) desenvolvimento adequado ao nível etário, (4) ausência de necessidades educativas especiais e (5) integração na turma com o mesmo professor desde o início do ano lectivo. Pretendeu-se ainda obter um número equilibrado de participantes de ambos os sexos e com níveis de escolaridade dos pais relativamente semelhantes.

Quanto à escolaridade dos pais, as mães apresentaram uma média de 9,5 anos de escolaridade $(D P=4,53$; sendo o mínimo de 2 e o máximo de 17 anos), e os pais uma média de 9,6 anos ( $D P=4,13$; mínimo de 3 e máximo de 17 anos). Estas duas variáveis encontram-se correlacionadas de forma positiva e estatisticamente significativa $(r=0,66, p<0,001)$, sugerindo que pais e mães tendem a apresentar níveis de escolaridade próximos. Esta correlação positiva, bem como o facto de a escolaridade da mãe surgir como uma das variáveis com maior valor preditivo na investigação sobre parentalidade (Hoff et al., 2002), levou-nos a considerar a variável escolaridade das mães como indicadora do nível socio-económico da família.

Como participantes deste estudo colaboraram ainda as dez professoras, responsáveis pelas turmas onde as crianças estavam integradas.

\section{Instrumentos}

O Questionário de Caracterização da Criança e do Contexto Familiar, preenchido pelas professoras, permitiu obter uma caracterização das crianças (por exemplo, data de nascimento e número de retenções) e dos seus agregados familiares (por exemplo, escolaridade dos pais).

A Escala de Avaliação do Conhecimento Emocional (EACE, Alves, 2006; Alves, Cruz, Duarte \& Martins, 2008) constitui a versão portuguesa, traduzida e adaptada da Assessment of Children's Emotion Skills (ACES, Shultz et al., 2004). Este instrumento, aplicado no contexto de uma entrevista à criança, é constituído por três subescalas - Expressões Faciais, Situações Emocionais e Comportamentos Emocionais - que permitem estudar o conhecimento que a criança tem de cinco sentimentos: alegria (alegre), tristeza (triste), zanga (zangado), medo (assustado) e normal ("não sentir nada em especial").

A subescala Expressões Faciais é constituída por 20 itens (quatro itens relativos a cada um dos cinco sentimentos) e analisa a capacidade das crianças para nomearem as expressões faciais. Para tal, são utilizadas 20 fotografias de crianças de idade escolar, manifestando diferentes expressões emocionais, face às quais a criança entrevistada deverá decidir qual o senti- 
mento da criança representada na fotografia, de entre as seguintes cinco alternativas atrás mencionadas.

A subescala Situações Emocionais é constituída por 15 vinhetas (três para cada um dos cinco sentimentos), face às quais a criança entrevistada terá que decidir como é que a criança protagonista se está a sentir. Por exemplo, no item 1 "O Alexandre fez um cartão bonito para o seu amigo João. O João gostou muito do cartão. Achas que o Alexandre se sente contente, triste, zangado, assustado ou normal?", é esperado que a criança reconheça na situação apresentada o sentimento de alegria.

Na subescala Comportamentos Emocionais cada criança, perante cada uma das 15 vinhetas (igualmente três para cada um dos cinco sentimentos), terá de identificar como é que a criança protagonista se está a sentir. Por exemplo, no item 1 "O João não tem vontade de jogar à bola no recreio, fica sentado sozinho. Achas que o João se sente, contente, triste, zangado, assustado ou normal? " é esperado que a criança reconheça o sentimento de tristeza.

Em cada subescala é calculado o total de respostas correctas para cada um dos cinco sentimentos. Para além desta pontuação, podemos ainda obter uma nota total da Percepção Emocional Correcta (PEC), que reflecte o número de vezes que cada criança respondeu correctamente, ao longo das três sub-escalas, aos itens relacionados com a alegria, tristeza, zanga e medo. Tal como sugerido por Schultz e colaboradores (2004), o cálculo da PEC, implicou a padronização prévia do total de respostas correctas apresentadas em cada uma das subescalas. Assim, para cada um dos cinco sentimentos existem 10 itens (quatro na sub-escala Expressões Faciais, três na sub-escala Situações Emocionais e três na sub-escala Comportamentos Emocionais). De acordo com os autores, a consistência interna da PEC é de 0,68 (alfa de Cronbach), ligeiramente inferior à obtida neste estudo $(0,70)$ e num estudo realizado por nós com crianças de idade pré-escolar (0,74; Alves et al., 2008).

As Escalas de Avaliação da Competência Social (Lemos \& Meneses, 2002) constituem a versão Portuguesa, traduzida e adaptada do Social Skills Rating System (Greshan \& Elliot, 1990), relativamente ao Ensino Básico. Os resultados encontrados para a população Portuguesa apoiam uma concepção multidimensional da competência social e garantem a validade da informação recolhida (Lemos \& Meneses, 2002). Este instrumento integra três escalas: Habilidades Sociais (30 itens), Problemas de Comportamento (18 itens) e Competência Académica (9 itens). As Habilidades Sociais incluem três sub-escalas: Cooperação (10 itens; e.g., "Realiza os trabalhos escolares de forma cuidada"), Auto-controle (10 itens; e.g., "Quando os colegas implicam com ele, reage adequadamente") e Asserção (10 itens; e.g., "Convida os colegas para actividades"). Os Problemas de Comportamento incluem as sub-escalas Problemas Internalizados (6 itens; e.g., "Isola-se"), Problemas Externalizados (7 itens; e.g., "Ameaça e goza os colegas") e Hiperactividade 
(5 itens; e.g., "Distrai-se facilmente”). A Competência Académica pretende avaliar o desempenho na leitura e na matemática, a motivação, o funcionamento cognitivo e o suporte parental. Enquanto que nas escalas Habilidades Sociais e Problemas de Comportamento, o professor deve sinalizar a frequência do comportamento do aluno em três pontos (1 - "nunca"; 2 - "às vezes"; 3 - "muitas vezes"), na Competência Académica é utilizada uma escala de cinco pontos (1 - "muito baixo"; 5 - "muito elevado"). De realçar que esta escala tem vindo a ser utilizada em Portugal em estudos recentes (Alves, 2006; Alves et al., 2008; Lemos \& Meneses, 2002; Lima, Teixeira, Serôdio \& Cruz, 2008) e apresenta uma consistência interna elevada (coeficientes alfa de Cronbach variam entre 0,90 e 0,98), possibilitando uma avaliação consistente da componente comportamental da competência social. De forma coerente com estes resultados, neste estudo a consistência interna das escalas Habilidades Sociais, Problemas de Comportamento e Competência Académica é de 0,96; 0,90 e 0,98, respectivamente.

Nesta investigação foi apenas utilizada a escala de habilidades sociais, dada o interesse específico pelo estudo do efeito mediador desta variável.

A aceitação pelos pares foi avaliada através de técnicas sociométricas. Estes procedimentos têm vindo a ser amplamente utilizados na investigação, permitindo medir e compreender a configuração socio-afectiva do relacionamento entre pares (Terry, 2002).

Depois da aplicação do questionário em cada turma, foi efectuada a codificação dos dados, tendo em vista a sua introdução no programa SOCIOM3 (Pio Abreu, 2002). Este programa fornece uma matriz sociométrica para cada turma, que inclui o número de rejeições reais recebidas pelo participante $(n)$, o número de preferências reais recebidas pelo participante $(p)$, bem como o valor médio e os valores extremos das pontuações de preferência e de rejeição.

De entre os vários modelos sociométricos, optámos pelo modelo padronizado de Coie, Dodge e Coppotelli (1982), que permite comparar grupos de tamanhos diferentes, como os envolvidos neste estudo. A aplicação deste modelo implicou a padronização das escolhas positivas ou preferências $(p)$ e negativas ou rejeições $(n)$, criando-se assim duas novas variáveis, $Z p$ e $Z n$. Estas foram posteriormente utilizadas no cálculo da preferência social, indiciadora da popularidade da criança, que corresponde à diferença entre o $Z p$ e $Z n$ e expressa a aceitação da criança no seu grupo de pares, designada neste estudo por aceitação pelos pares.

\section{Procedimento}

Obtido o consentimento informado das escolas e dos encarregados de educação (apenas 3\% recusaram autorizar os filhos a participar), procedemos à selecção dos participantes, em função dos critérios previamente referidos. 
A recolha de dados decorreu no final do terceiro período lectivo, nas escolas frequentadas por cada uma das crianças, implicando três momentos diferenciados. Num primeiro momento, as professoras preencheram, para cada criança, o Questionário de Caracterização da Criança e do Contexto Familiar e as Escalas de Competência Social.

Num segundo momento, procedeu-se à aplicação do teste sociométrico a todos os alunos de cada turma, evitando situações de exclusão. Esta aplicação ocorreu no final do ano lectivo, altura em que se espera que a organização social, decorrente dos relacionamentos entre os elementos de cada turma, esteja definida (Moreno, 1934, p. 114). O teste sociométrico foi aplicado na presença da professora, respondendo-se às dúvidas apresentadas pelas crianças; foi-lhes ainda pedido que não revelassem o sentido das suas escolhas, sensibilizando-as para a importância das normas de confidencialidade. Não foram verificadas dificuldades de resposta por parte das crianças, nem se registou qualquer resposta inválida (por exemplo, "não sei", "são todos") ou questionário em branco.

Num terceiro momento, que decorreu numa sala disponibilizada na escola para o efeito, procedeu-se à administração individual da EACE, que demorou em média 10 minutos. De realçar que as crianças participaram de forma voluntária após a apresentação da tarefa, mostraram-se envolvidas e interessadas, não revelando qualquer resistência aberta.

\section{Resultados}

\section{Análises Descritivas}

Relativamente ao conhecimento emocional, verifica-se que os participantes apresentam uma percentagem de sucesso mais elevada nos itens que envolvem as Expressões Faciais (61\%) do que nos itens relativos às Situações Emocionais (56\%) e aos Comportamentos Emocionais (53\%). Estes dados são consistentes com as médias apresentadas no Quadro 1. A análise dos resultados em função do sexo da criança não revelou quaisquer diferenças significativas $(t=0,72$, n.s., $t=0,63$, n.s., $t=0,91$, n.s., respectivamente para as Expressões Faciais, Situações Emocionais e Comportamentos Emocionais).

Em termos das habilidades sociais, é na sub-escala de auto-controlo que se verifica a média mais baixa (cf. Quadro 1). A análise dos resultados em função do sexo mostra que os rapazes e as raparigas apenas se diferenciaram de forma significativa na sub-escala cooperação, apresentando-se as raparigas como mais cooperantes $(t=1,512 ; p<0,05)$.

No que respeita à aceitação pelos pares (cf. Quadro 1), verificamos que esta não difere de forma significativa entre os rapazes e as raparigas e os resultados obtidos aproximam-se dos apresentados na literatura (Coie et al., 1982). 
Quadro 1. Média, desvio-padrão e amplitude do conhecimento emocional, das habilidades sociais e da aceitação pelos pares.

\begin{tabular}{llllc}
\hline & & Média & DP & Amplitude \\
\hline Conhecimento & Expressões & 12,20 & 2,20 & $0-20$ \\
emocional & Situações & 8,40 & 2,00 & $0-15$ \\
& Comportamentos & 8,00 & 2,20 & $0-15$ \\
& PEC & 28,70 & 5,70 & $0-50$ \\
\hline Habilidades & Cooperação & 13,70 & 5,5 & $0-30$ \\
sociais & Auto-controlo & 9,60 & 4,1 & $0-30$ \\
& Asserção & 12,60 & 5,0 & $0-30$ \\
& TOTAL & 35,90 & 13,2 & $0-90$ \\
\hline Aceitação pelos & Aceitação pelos pares & 0,14 & 0,9 & na \\
\hline
\end{tabular}

Nota: na - não aplicável

No Quadro 2 são apresentadas as correlações entre as variáveis. Verifica-se que o conhecimento emocional e as habilidades sociais se correlacionam significativamente entre si e com a aceitação pelos pares. Relativamente à escolaridade das mães, esta mostra-se moderadamente correlacionada com o conhecimento emocional, as habilidades sociais e a aceitação pelos pares.

Depois de controlada a escolaridade das mães, constata-se que a correlação entre o conhecimento emocional e a aceitação pelos pares diminui, mas mantém-se estatisticamente significativa ( $r$ parcial $=0,20 ; p<0,05)$. O mesmo acontece com a correlação entre as habilidades sociais e a aceitação pelos pares $(r$ parcial $=0,29 ; p<0,01)$. Também a correlação entre o conhecimento emocional e as habilidades sociais diminui quando se controla a escolaridade das mães, mantendo-se o nível de significância estatística $(r$ parcial $=0,24$; $p<0,05)$. Assim, tal como seria de esperar, o conhecimento emocional apresenta associações positivas e estatisticamente significativas com as habilidades sociais, por um lado, e com a aceitação pelos pares, por outro.

\section{Estimação do Modelo de Mediação}

Seguidamente testámos os efeitos de mediação de acordo com a hipótese acima delineada e seguindo o modelo proposto por Baron e Kenny (1986). De acordo com este modelo, uma vez constatada a relação entre o preditor (ou variável independente) e a variável dependente, uma terceira 
variável poderá funcionar como mediadora se contribuir para a relação verificada. Teremos então que assegurar o cumprimento de três condições: "a) variações nos níveis da variável independente contribuem significativamente para variações nas variáveis presumivelmente mediadoras; $b$ ) variações nos mediadores contribuem significativamente para variações na variável dependente" (Baron \& Kenny, 1986, p. 1176), e ainda, c) efectuando a regressão conjunta da variável independente e da variável mediadora na variável dependente, o efeito da variável independente na variável dependente diminui.

Quadro 2. Correlações entre as variáveis do estudo

\begin{tabular}{|c|c|c|c|c|c|c|c|c|c|}
\hline & 1 & 2 & 3 & 4 & 5 & 6 & 7 & 8 & 9 \\
\hline \multicolumn{10}{|l|}{$\begin{array}{l}\text { 1. Escolaridade } \\
\text { das mães }\end{array}$} \\
\hline 2.Expressões & $0,20 * *$ & & & & & & & & \\
\hline 3. Situações & $0,26^{* *}$ & $0,41 * * *$ & & & & & & & \\
\hline \multicolumn{10}{|c|}{ 4.Comportamentos $0,37 * * 0,45 * * * 0,50 * * *$} \\
\hline $\begin{array}{l}\text { 5. Percepção Emo- } \\
\text { cional Correcta }\end{array}$ & $0,34 * *$ & $0,78 * *$ & $0,80 * *$ & $0.82 * *$ & & & & & \\
\hline 6. Cooperação & $0,32 * *$ & $0,31 * *$ & $0,31 * *$ & $0,34 * *$ & $0,40 * *$ & & & & \\
\hline 7. Auto-controlo & $0,32 * *$ & 0,06 & $0,19^{*}$ & $0,21 *$ & $0,19 *$ & $0,78 * * *$ & & & \\
\hline 8. Asserção & $0,45^{* *}$ & 0,17 & $0,28 * *$ & $0,31 * *$ & $0,32 * *$ & $0,69 * * *$ & $0,74 * * *$ & & \\
\hline $\begin{array}{l}\text { 9. Habilidades } \\
\text { sociais }\end{array}$ & $0,41 * *$ & 0,21 & $0,29 * *$ & $0,32 * *($ & $0,34 * *$ & $0,92 * * *$ & $0,91 * * *$ & $0,89 * * *$ & \\
\hline $\begin{array}{l}\text { 10. Aceitação pelos } \\
\text { pares }\end{array}$ & $0,26 * *$ & $0,19 *$ & $0,21 *$ & $0,25 * *$ & $0,27 * *$ & $0,45 * * *$ & $0,29 * *$ & $0,23 * *$ & $0,36 * * *$ \\
\hline
\end{tabular}

Nota: $p<0,05^{*} ; p<0,01^{* *} ; p<0,001^{* * *}$

No modelo que pretendemos testar, o conhecimento emocional é a variável preditora, a aceitação pelos pares a variável dependente e as habilidades sociais a variável mediadora desta relação (cf. Figura 1). Para testar o efeito da mediação é necessário estimar três equações de regressão, tendo-se constatado que: a) o conhecimento emocional (preditor) contribui significativamente para a variância das habilidades sociais (hipotético mediador) na equação $1(\beta=0,28 p<0,001)$; b) o conhecimento emocional (preditor) contribui significativamente para a variância da aceitação pelos pares na equação $2(\beta=0,23, p<0,008)$; c) as habilidades sociais contribuem significativa- 
mente para a variância da aceitação pelos pares, depois de removida a variância do conhecimento emocional, na equação $3\left(R^{2}=0,13, \beta=0,32\right.$, $p<0,001)$. A relação significativa existente entre o conhecimento emocional e a aceitação pelos pares expressa na equação 2 decresce para valores não significativos $\left(R^{2}=0,15 \beta=0,14, p>0,10\right)$, confirmando o efeito mediador das habilidades sociais (Baron \& Kenny, 1986).

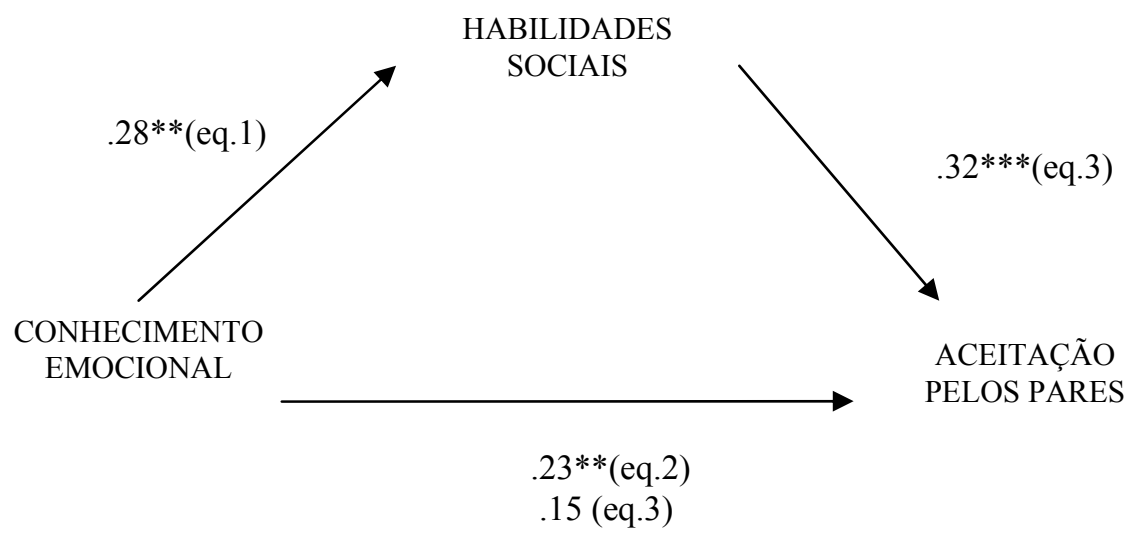

Nota: $* * p<0,01 ; * * * p<0,001$

Figura 1: Mediação das habilidades sociais na relação entre conhecimento emocional e a aceitação pelos pares.

O teste de Sobel (1982) permitiu verificar que o efeito mediador das habilidades sociais na relação entre o conhecimento emocional e a aceitação pelos pares é estatisticamente significativo $(Z=3,47, p<0,01)$, indicando que as habilidades sociais contribuem para explicar o efeito preditor do conhecimento emocional da criança na sua aceitação pelos pares.

Em síntese, as análises acima apresentadas evidenciam a mediação das habilidades sociais na relação entre o conhecimento emocional e a aceitação pelos pares.

\section{Discussão}

Este estudo pretendeu investigar a contribuição do conhecimento emocional para a aceitação pelos pares, procurando identificar em que medida as habilidades sociais podem contribuir para explicar esta relação. De 
realçar que as análises estatísticas realizadas possuem um cariz correlacional, não permitindo inferências de causalidade.

Os resultados deste estudo mostram que o conhecimento emocional e as habilidades sociais contribuem para a aceitação pelos pares em idade escolar (Mostow et al., 2002). As habilidades sociais assumem um papel mediador na relação entre o conhecimento emocional e a aceitação pelos pares, ou seja, o poder preditivo do conhecimento emocional na aceitação pelos pares é explicado pelas habilidades sociais da criança. Este resultado é consistente com o modelo de processamento da informação social, na reformulação proposta por Lemerise e Arsenio (2002), no sentido de integrar a dimensão emocional com a dimensão social nas diferentes etapas do processamento. $\mathrm{O}$ conhecimento emocional revela-se especialmente relevante nas duas primeiras etapas de codificação e interpretação da informação, ou seja, no reconhecimento e nomeação correcta de estímulos que desencadeiam expressões e comportamentos emocionais. As crianças com mais habilidades sociais utilizam de forma mais adequada a informação emocional no contexto das interacções entre pares, conjugando-se assim estes dois factores no sentido de promover um estatuto de maior aceitação (ou popularidade) entre pares.

Relativamente ao papel dos factores individuais, os resultados reflectem a ausência de diferenças entre sexos no conhecimento emocional e nas habilidades sociais das crianças, à excepção da cooperação que se revela superior nas raparigas, à semelhança do encontrado por outros autores (Denham, 1998; Eisenberg \& Fabes, 1998). Pelo contrário, e consistentemente com a literatura (cf. Hoff et al., 2002), o funcionamento social e emocional das crianças varia em função da escolaridade das mães, sendo que esta variável contribui para explicar apenas parcialmente as relações que se estabelecem entre o conhecimento emocional, as habilidades sociais e a aceitação pelos pares.

Conscientes de que o comportamento que as crianças exibem na escola é um elemento crucial de todo o seu funcionamento socio-emocional, optámos por avaliar o fenómeno da aceitação social neste contexto primordial do desenvolvimento. Não só porque é na escola que as crianças passam a maior parte do seu tempo, mas também porque este é um contexto privilegiado para aprender e praticar competências sociais e desenvolver relações (formais e informais) com pares e adultos.

Embora nos tenhamos centrado apenas num contexto, no seio deste foram consideradas diferentes fontes de informação: os professores, os pares e a própria criança. Assim, a avaliação das habilidades sociais foi feita junto dos professores, a aceitação pelos pares junto destes mesmos e o conhecimento emocional junto da criança. A avaliação efectuada pelos professores caracteriza o comportamento social da criança no contexto escolar e as medidas sociométricas manifestam o sentimento colectivo relativamente à 
criança (Almeida, 1997). Assim, a primeira permite-nos perceber como é que o comportamento da criança é percepcionado pelos professores e a segunda indica-nos a percepção desse comportamento junto dos pares, constituindo-se como medidas complementares.

Apesar de a escola ser um contexto muito importante para as crianças de idade escolar, as suas interacções também ocorrem noutros contextos. A vida de um número considerável de crianças é substancialmente enriquecida pela participação em actividades fora do contexto escolar, com ganhos evidentes na realização académica e na diminuição do abandono escolar (Larson, Jarrett, Hansen, Pearce, Sullivan, Walker et al., 2004). Sublinhamos assim uma primeira limitação deste estudo, sendo plausível que um número razoável de crianças apresente diferentes níveis de popularidade no contexto escolar, no clube desportivo ou na vizinhança. Futuras investigações deverão efectuar o estudo da aceitação social "fora" do contexto escolar.

Uma segunda limitação deste trabalho refere-se ao limitado número de determinantes da aceitação pelos pares considerado. O modelo do processamento da informação reformulado por Lemerise e Arsenio (2000) salienta o papel da dimensão emocional ao longo das diversas etapas do processamento da informação, remetendo-nos para a importância de considerar a competência emocional de uma forma mais ampla. Ou seja, para além do conhecimento emocional, é fundamental considerar-se em futuros estudos a regulação emocional e a expressão emocional (Eisenberg, Fabes, Guthrie \& Reiser, 2000). Da mesma forma, neste modelo é também sublinhado o papel das experiências anteriores e das predisposições temperamentais, nomeadamente da emocionalidade. Assim, parece-nos que a integração das interacções parentais (Yap, Allen, Love \& Katz, 2008) e do temperamento (Rothbart \& Putnam, 2002) em trabalhos futuros que visem o estudo da aceitação pelos pares, será fundamental na construção de um modelo mais amplo dos preditores da aceitação pelos pares.

Parece-nos que o conhecimento dos processos envolvidos na qualidade das interacções com pares é especialmente relevante na determinação de uma trajectória desenvolvimental ajustada. A longo prazo, a recorrência de percepções e interpretações parciais ou incorrectas das pistas emocionais pode impedir o normal desenvolvimento socio-emocional e comprometer o ajustamento social da criança (Izard et al., 2001). O conhecimento emocional parece afectar a interpretação do estímulo social e consequentemente as tomadas de decisão que as crianças desenvolvem nas situações sociais. (Izard, 1993; Lemerise \& Arsenio, 2000) Estes possíveis efeitos poderão ocorrer de forma directa, na interpretação incorrecta das emoções dos outros, ou indirectamente, quando as interpretações erróneas levam à evocação e à experimentação de emoções desadequadas, produzindo um conjunto de erros no processamento da informação social (Fine et al., 2003). 
Esperamos que o estudo aqui apresentado contribua para a construção de um conhecimento que alicerce a planificação de programas de intervenção universal com vista à promoção do comportamento pró-social e à prevenção dos problemas de comportamento. Tal como referem Fine e colaboradores (2003), estes programas deverão ser implementados precocemente e incluir oportunidades para a criança promover o seu conhecimento emocional. Este parece constituir a base de outras competências sociais essenciais e surge como um dos principais preditores da competência académica e do comportamento social (Izard et al., 2001; Machado et al., 2008).

\section{Referências}

Almeida, A. M. T (1997). As relações entre pares em idade escolar. Um estudo de avaliação da competência social pelo método de Q-sort. Tese de Doutoramento não publicada, Instituto de Estudos da Criança, Universidade do Minho.

Arsenio, W. F., Cooperman, S., \& Lover, A. (2000). Affective predictors of preschoolers aggression and peer acceptance: Direct and indirect effects. Developmental Psychology, 36, 438-448.

Arsenio, W. F., \& Lover, A. (1995). Children's conceptions of sociomoral affect: Happy victimizers, mixed emotions, and other expectancies. In M. Killen \& D. Hart (Eds.) Morality in everyday life: developmental perspectives (pp. 87-128). Cambridge: Cambridge University Press.

Alves, D. (2006). O emocional e o social na idade escolar: Uma abordagem dos preditores da aceitação pelos pares. Tese de Mestrado não publicada, Faculdade de Psicologia e de Ciências da Educação, Universidade do Porto.

Alves, D., Cruz, O., Duarte, C., \& Martins, R. (2008). Escala de Avaliação do Conhecimento Emocional (EACE). In A. P. Noronha, C. Machado, L. Almeida, M. Gonçalves, S. Martins \& V. Ramalho (Coords.), Avaliação psicológica: Formas e contextos - Actas. Braga: Psiquilibrios Edições. (CD-ROM, item $\left.\mathrm{n}^{\circ} 9789899552265\right)$

Baron, R. M., \& Kenny, D. A. (1986). The moderator-mediator variable distinction in social psychological research: Conceptual, strategic and statistical considerations. Journal of Personality and Social Psychology, 51, 1173-1182.

Cassidy, J., Parke, R. D., Butkovsky, L., \& Braungart, J. M. (1992). Family-peer connections: The roles of emotional expressiveness within the family and children's understanding of emotions. Child Development, 63, 603-618.

Coie, J. D., Dodge, K. A., \& Kupersmidt, J. B. (1990). Peer group behaviour and social status. In S. Asher \& J. Coie (Eds.), Peer rejection in childhood (pp. 17-59). Cambridge: Cambridge University Press.

Coie, J. D., Dodge, K., \& Coppotelli, H. A. (1982). Dimensions and types of social status: A cross-age perspective. Developmental Psychology, 18, 557-569. 
Crick, N. R., \& Dodge, K. A. (1994). A review and reformulation of social information-processing mechanisms in children's social adjustment. Psychological Bulletin, 115, 74-101.

Denham, S. A. (1998). Emotional development in young children. New York: Guilford Press.

Denham, S. A., McKinley, M., Couchoud, E. A., \& Holt, R. (1990). Emotional and behavioural predictors of preschool peer ratings. Child Development, 61, 1145-1152 .

Denham, S. A., \& Holt, R. W. (1993). Preschoolers' likability as cause or consequence of their social behavior. Developmental Psychology, 29, 271-275.

Dodge, K. A. (1983). Behavioral antecedents of peer social status. Child Development, 54, 1386-1399.

Dodge, K. A., \& Feldman, E. (1990). Issues in social cognition and sociometric status. In S. R. Asher \& J. D. Coie (Eds.), Peer rejection in childhood (pp. 119-155). New York: Cambridge Studies.

Dodge, K. A., Lansford, J. E., Burks, V. S., Bates, J. E., Pettit, G. S., Fontaine, R., \& Price, J. M. (2003). Peer rejection and social information-processing factors in the development of aggressive behaviour problems in children. Child Development, 74, 374-393.

Eisenberg, N., \& Fabes, R. (1998). Prosocial development. In W. Damon (Series Ed.), \& N. Eisenberg (Ed.), Handbook of child psychology: Social, emotional, and personality development (Vol. 3, pp. 701-778). New York: Wiley.

Eisenberg, N., Fabes, R. A., Guthrie, I. K., \& Reiser, M. (2000). Dispositional emotionality and regulation: Their role in predicting quality of social functioning. Journal of Personality and Social Psychology, 78, 136-157.

Eisenberg, N., Murphy, B. C., \& Shepard, S. (1997). The development of empathic accuracy. In W. Ickes (Ed.), Empathic accuracy (pp. 73-116). New York: The Guilford Press.

Fine, S. E., Izard, C. E., Mostow, A. J., Trentacosta, C. J., \& Ackerman, B. P. (2003). First grade emotion knowledge as a predictor of fifth grade self-reported internalizing behaviours in children from economically disadvantaged families. Development and Psychopathology, 15, 331-342.

Gresham, F. M., \& Elliott, S. N. (1990). Social skills rating system manual. Circle Pines, MN: American Guidance Service.

Hoff, E., Laursen, B., \& Tardif, T. (2002). Socioeconomic status and parenting. In M. Bornstein (Ed.), Handbook of parenting, Vol.2: Biology and ecology of parenting (pp. 231-252). Mahwah: Lawrence Erlbaum Associates, Publishers.

Hobson, P. (1993). The emotional origins of social understanding. Philosophical Psychology, 6, 227-249.

Izard, C. E. (1971). The face of emotion. New York: Appleton-Century-Croft.

Izard, C. E. (1991). The psychology of emotions. New York: Plenum.

Izard, C. E. (1993). Four systems for emotion activation: Cognitive and noncognitive processes. Psychological Review, 100, 68-90. 
Izard, C. E., Ackerman, B. P. (2004). Motivational, organization and regulatory functions of discrete emotions. In M. Lewis \& J. M. Haviland-Jones (Eds.), Handbook of emotions (pp. 253-264). New York: The Guilford Press.

Izard, C. E., Fine, S. E., Schultz, D., Mostow, A. J., Ackerman, B. P., \& Youngstrom, E. (2001). Emotion knowledge as a predictor of social behavior and academic competence in children at risk. Psychological Science, 12, 18-23.

Lemerise, E. A., \& Arsenio, W. F. (2000). An integrated model of emotion processes and cognition in social information processing. Child Development, 71, $107-118$.

Lemos, M., \& Meneses, H. (2002). A avaliação da competência social: Versão portuguesa da forma para professores do SSRS. Psicologia: Teoria e Pesquisa, 18, 267-274.

Lima, A., Teixeira, V., Serôdio, R., \& Cruz, O. (2008). Escala de problemas de comportamento em crianças de idade escolar. In A. P. Noronha, C. Machado, L. Almeida, M. Gonçalves, S. Martins \& V. Ramalho (Coords.), Avaliação Psicológica: Formas e Contextos - Actas. Braga: Psiquilibrios Edições. (CD-ROM, item nº789899552265)

Larson, R., Jarrett, R., Hansen, D., Pearce, N., Sullivan, P., Walker, K., Watkins, N., \& Wood, D. (2004) Organized youth activities as contexts for positive development. In A. Linley \& S. Joseph (Eds.), International handbook of positive psychology in practice: From research to application (pp. 540-560). Hoboken: John Wiley \& Sons, Inc.

Machado, P., Veríssimo, M., Torres, N., Peceguina, I., Santos, A. J., \& Rolão, R. (2008). Relações entre o conhecimento das emoções, as competências académicas, as competências sociais e a aceitação pelos pares. Análise Psicológica, 3, 463-478.

Moreno, J. L. (1934). Who shall survive? A new approach to the problem of human interrelations. Washington, D. C: Nervous and Mental Diseases Publishing Co.

Mostow, A., Izard, C. E., Fine, S., \& Trentacosta, C. J. (2002). Modeling emotional, cognitive, and behavioral predictors of peer acceptance. Child Development, 73, 1775-1787.

Pio Abreu, J. L. (2002). SOCIOMETRIA: Trabalhar com o programa SOCIOM3. Coimbra: Hospital da Universidade de Coimbra.

Rothbart, M. K., \& Putnam, S. P. (2002). Temperament and socialization. In L. Pulkinnen \& A. Caspi (Eds.), Paths to successful development: Personality in the life course (pp. 19-45). Cambridge: Cambridge University Press.

Rubin, K. H., Bukowski, W., \& Parker, J. G. (2006). Peer interactions, relationships, and groups. In W. Damon, R. M. Lerner \& N. Eisenberg (Eds.), Handbook of child psychology, Vol. 3: Social, emotional and personality development (pp. 571-645). New York: Wiley.

Sobel, M. E. (1982). Asymptotic confidence intervals for indirect effects in structural equation models. In S. Leinhardt (Ed.), Sociological methodology (pp. 290-312). Washington DC: American Sociological Association. 
Schultz, D., Izard, C. E., \& Ackerman, B. P. (2000). Children's anger attribution bias: Relations to family adjustment and social adjustment. Social Development, 9, 284-301.

Schultz, D., Izard, C. E., \& Bear, G. (2004). Children emotion processing: Relations to emotionality and aggression. Development and Psychopathology, 16, 371-387 .

Terry, R. (2000). Recent advances in measurement theory and the use of sociometric techniques. In A. H. N. Cillesen \& W. M. Bukowski (Eds.), New directions for child and adolescent development: Vol. 88. Recent advances in the measurement of acceptance and rejection in the peer system (pp. 27-53). San Francisco: Jossey-Bass.

Yap, M. B., Allen, N. B., Love, C., \& Katz, L. F. (2008). Maternal meta-emotion philosophy and socialization of adolescent affect: The moderating role of adolescent temperament. Journal of Family Psychology, 22, 688-700.

Zettergren, P. (2005). Childhood peer status as predictor of midadolescence peer situation and social adjustment. Psychology in Schools, 42, 745-757. 